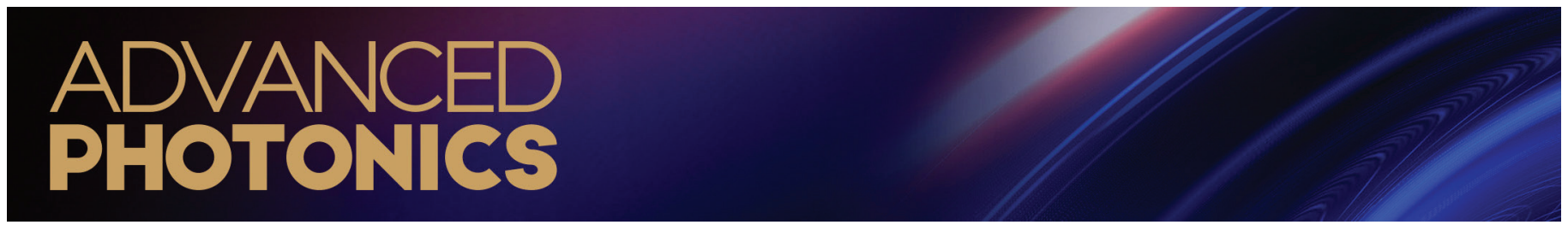

\title{
Perovskite polariton parametric oscillator
}

\author{
Jinqi Wu, ${ }^{a}$ Rui Su, ${ }^{a, *}$ Antonio Fieramosca $\odot,{ }^{a}$ Sanjib Ghosh, ${ }^{a}$ Jiaxin Zhao, ${ }^{a}$ Timothy C. H. Liew, ${ }^{\mathrm{a}, \mathrm{b}, \star}$ and \\ Qihua Xiong ${ }^{c, d, e, *}$ \\ ${ }^{a}$ Nanyang Technological University, School of Physical and Mathematical Sciences, Division of Physics and Applied Physics, Singapore \\ 'MajuLab, International Joint Research Unit UMI 3654, CNRS, Université Côte d'Azur, Sorbonne Université, National University of Singapore, \\ Nanyang Technological University, Singapore \\ 'Tsinghua University, State Key Laboratory of Low-Dimensional Quantum Physics, Department of Physics, Beijing, China \\ ${ }^{\mathrm{d} B e i j i n g ~ A c a d e m y ~ o f ~ Q u a n t u m ~ I n f o r m a t i o n ~ S c i e n c e s, ~ B e i j i n g, ~ C h i n a ~}$ \\ ${ }^{\mathrm{e}}$ Tsinghua University, Beijing Innovation Center for Future Chips, Beijing, China
}

\begin{abstract}
Optical parametric oscillators (OPOs) have been widely applied in spectroscopy, squeezed light, and correlated photons, as well as quantum information. Conventional OPOs usually suffer from a high power threshold limited by weak high-order nonlinearity in traditional pure photonic systems. Alternatively, polaritonic systems based on hybridized exciton-photon quasi-particles exhibit enhanced optical nonlinearity by dressing photons with excitons, ensuring highly nonlinear operations with low power consumption. We report an on-chip perovskite polariton parametric oscillator with a low threshold. Under the resonant excitation at a range of angles, the signal at the ground state is obtained, emerging from the polariton-polariton interactions at room temperature. Our results advocate a practical way toward integrated nonlinear polaritonic devices with low thresholds.
\end{abstract}

Keywords: inorganic perovskite semiconductors; exciton-polaritons; parametric oscillators; four-wave mixing.

Received Jul. 29, 2021; revised manuscript received Sep. 22, 2021; accepted for publication Oct. 7, 2021; published online Oct. 31, 2021.

(C) The Authors. Published by SPIE and CLP under a Creative Commons Attribution 4.0 International License. Distribution or reproduction of this work in whole or in part requires full attribution of the original publication, including its DOI.

[DOI: 10.1117/1.AP.3.5.055003]

\section{Introduction}

Optical parametric oscillators (OPOs) convert a pump frequency $\omega_{p}$ into signal $\omega_{s}$ and idler $\omega_{i}$ frequencies, satisfying the energy conservation law (with the second-order nonlinearity, $\omega_{p}=$ $\omega_{i}+\omega_{s}$; with the third-order nonlinearity, $2 \omega_{p}=\omega_{i}+\omega_{s}$ ). These indispensable devices have been widely used in creating tunable and coherent light for the application of spectroscopy. ${ }^{1}$ In addition, OPOs have attracted considerable interest in other fields such as the generation of squeezed light, ${ }^{2,3}$ correlated photons, ${ }^{4-6}$ and quantum information including quantum random number generation and Ising machines. ${ }^{7}$ In traditional systems, OPOs usually require noncentrosymmetric crystals (secondorder nonlinearity) embedded in an external resonator, which is resonant with the pump wavelength as well as the wavelength of generated signal and idler states. Such generated signal and idler states can be further tuned by controlling the phase matching condition via changing the temperature or angle of the

*Address all correspondence to Qihua Xiong, Qihua_xiong@tsinghua.edu.cn; Timothy C. H. Liew, Timothyliew@ntu.edu.sg; Rui Su, surui@ntu.edu.sg. nonlinear crystal or tuning the pump wavelength. ${ }^{8}$ However, such traditional OPOs usually suffer from a high power threshold, ${ }^{8}$ since anisotropic crystals require stringent birefringence phase matching requirements and usually exhibit limited high-order nonlinearity. ${ }^{9}$ Moreover, crystals in centrosymmetric space groups such as all-inorganic cesium lead halide perovskites $\left(\mathrm{CsPbBr}_{3}\right)$ need to break inversion-symmetry by anion substitution or other specific complex processes. ${ }^{10-12}$ Despite the recent progresses achieved in various bulk whispering gallery mode-based OPOs, ${ }^{13-16}$ the threshold powers are still relatively large, particularly the chip-scale planar OPOs, ${ }^{8,17-19}$ which hinder the road toward integrated nonlinear photonic devices.

Alternatively, exciton-polaritons are hybrid quasi-particles resulting from the strong coupling between photons and excitons, inheriting a high nonlinearity from their excitonic component. The nonlinearity of such a polariton system is orders of magnitude stronger than that of traditional nonlinear optical systems, which opens a new way for nonlinear polaritonic devices. ${ }^{20-22}$ Thanks to S-shaped polariton dispersion, the idler at a high $k$ (wavevector) state and the signal at the ground state 
can be obtained when two polaritons are resonantly pumped at the inflection point, obeying the conservation law of both energy and momentum. ${ }^{23-25}$ In addition to this nondegenerate polariton oscillator geometry, in a system with multiple branches, two polaritons at the lowest energy of one polariton branch can scatter to momentum-degenerate states $^{26-28}$ or energy-degenerate states $^{29-32}$ at adjacent polariton branches. The large third-order polaritonic nonlinearity promotes the realization of polariton oscillators at lower threshold, which has been demonstrated in a variety of semiconductor microcavities. ${ }^{23-32}$ Compared with the conventional OPOs, the polaritonic configuration is more versatile and suitable for the realization of quantum correlation and parametric down-conversion toward miniaturized, integrated devices. ${ }^{22,28-30}$ So far, polariton oscillators in GaAs or CdTe-based quantum wells demand cryogenic temperatures due to the small exciton binding energy, whereas polariton oscillators have not been realized in organic microcavities due to the low nonlinearity of Frenkel excitons.

Halide perovskites with a wide structural diversity, tunable bandgap, and remarkable optical properties, have rapidly emerged as excellent semiconductors for photonic technologies ranging from light-emitting diodes ${ }^{33,34}$ and lasers ${ }^{35,36}$ to photodetectors. ${ }^{37}$ More recently, for engineering their applications in next-generation photonic devices, the nonlinear properties of perovskite attract tremendous interest. The versatile architecture of halide perovskites along with their scalable synthesis allows for structural and electronic engineering for nonlinear optical functionalities including OPOs, ultra-fast laser modulators, ${ }^{11,38}$ and $\mathrm{THz}$ generators/detectors. ${ }^{39,40}$ Due to the large exciton binding energy, perovskite microcavities can sustain robust exciton polaritons and polariton lasing with lower threshold at room temperature. ${ }^{41-44}$ Moreover, the strong nonlinear polariton-polariton interaction has been demonstrated in both 2D hybrid perovskites and 3D all-inorganic perovskites. ${ }^{32,45}$ Combining these advantages, halide perovskites provide a promising platform for nonlinear polaritonic devices with low thresholds. Although energy-degenerate parametric scattering has been reported in $3 \mathrm{D}$ all-inorganic perovskites, the fixed wavelength of the signal and idler is not adjustable. This limits some applications, specifically down conversion, as well as the possibility of reaching the quantum correlated regime, due to the presence of degenerate uncorrelated scattering processes. $^{46}$

Here, we demonstrate nondegenerate polariton parametric oscillators with low thresholds in all-inorganic $\mathrm{Cs} \mathrm{PbBr}_{3}$ microcavities at room temperature. This is achieved by taking advantage of the large third-order nonlinearity and S-shaped polariton dispersion of our on-chip perovskite microcavities.

\section{Methods}

\subsection{Perovskite Microcavity Fabrication}

The perovskite microcavity is formed by the central perovskite embedded in a bottom distributed Bragg reflector (DBR) and a top DBR. First, the all-inorganic $\mathrm{CsPbBr}_{3}$ single crystal film is grown on a mica substrate via chemical vapor deposition (CVD) as reported before. ${ }^{47,48}$ Subsequently, the perovskite is tape-transferred to the bottom DBR composed of 30.5 pairs of $\mathrm{TiO}_{2} / \mathrm{SiO}_{2}$, followed by the spin-coating of a PMMA spacer layer. Finally, another 7.5 pairs of $\mathrm{TiO}_{2} / \mathrm{SiO}_{2}$ are deposited by an electron beam evaporator as the top DBR.

\subsection{Optical Spectroscopy Characterizations}

The momentum-space PL mappings are measured by a homebuilt angle-resolved spectroscopy setup consisting of the excitation, transparent stage, and detection in order with Fourier optics at room temperature. In the linear regime, the perovskite microcavity is pumped by the continuous-wave (CW) laser $(457 \mathrm{~nm})$ at normal incidence with a spot size of $10 \mu \mathrm{m}^{2}$. In the nonlinear regime, the excitation source is a pulsed laser (pulse width: $6 \mathrm{~ns}$, repetition rate: $20 \mathrm{~Hz}$ ) with tunable wavelength. It goes through a $50 \times$ microscope objective $(\mathrm{NA}=0.75)$ to resonantly excite the microcavity (spot size $\sim 10 \mu \mathrm{m}^{2}$ ) with an adjustable angular range of $\pm 48.6 \mathrm{deg}$ at each wavelength. After excitation, the transmission from the perovskite microcavity is collected by a $100 \times$ microscope objective $(\mathrm{NA}=0.9)$ covering an angular range of $\pm 64.1 \mathrm{deg}\left(k_{x}=10.8 \mu \mathrm{m}^{-1}\right)$ and sent to the spectrometer (Horiba iHR550) equipped with a grating of 600 lines $\mathrm{mm}^{-1}$ and a liquid-nitrogen-cooled charge coupled device of $256 \times$ 1024 pixels.

\subsection{Theoretical Calculations}

Since our experimental condensates occur only at the lower branch of the polariton dispersion, we consider an effective dynamical equation for the condensate wavefunction $\Psi(\vec{r}, t)$, given by

$$
\begin{aligned}
i \hbar \dot{\Psi}(\vec{r}, t)= & {\left[(1-i \lambda) \hat{H}_{T}+V(\vec{r})-i \gamma\right.} \\
& \left.+\left(\alpha_{R}-i \alpha_{I}\right)|\Psi(\vec{r}, t)|^{2}\right] \Psi(\vec{r}, t)+F(\vec{r}),
\end{aligned}
$$

where $\hat{H}_{T}=\left[\hat{E}_{k}+E_{x}-\sqrt{\left(\hat{E}_{k}-E_{x}\right)^{2}+4 g^{2}}\right] / 2$ is the kinetic part of the Hamiltonian operator for the lower polariton (LP) branch, $\hat{E}_{k}=\hbar^{2} \hat{k}^{2} /\left(2 m_{c}\right)$ is the photonic part of the kinetic energy, $E_{x}$ is the exciton energy with respect to the lowest photon energy, $V(\vec{r})$ is a random potential representing the disorder in the system, and $F(\vec{r})=F_{0} e^{i \vec{k}_{p}, \vec{r}-r^{2} / A_{p}}$ is the coherent pump applied to the condensate. The parameter $\gamma$ is the decay strength due to a finite polariton lifetime, $\alpha_{R}$ is the nonlinear interaction strength, $\alpha_{I}$ is the nonlinear decay strength, $\lambda$ is a phenomenological parameter representing the energy relaxation, $m_{c}$ is the effective mass of the photonic mode, $g / \hbar$ is the Rabi frequency, $A_{p}$ is the effective pump area, $\vec{k}_{p}$ is the wavevector of the pump, and $F_{0}$ is a constant representing the strength of the pump. The energy dispersion is calculated using the formula

$I\left(k_{x}, E\right)=\int_{-\infty}^{\infty} \mathrm{d} \omega e^{-(E-\omega)^{2} / \Delta_{\omega}{ }^{2}}\left|\tilde{\Psi}\left(k_{x}, k_{y}=0, \omega\right)\right|^{2}$,

where $\Delta_{\omega}$ is the energy resolution in the dispersion, and $\tilde{\Psi}(\vec{k}, \omega)$ is the Fourier transform of the wavefunction $\Psi(\vec{r}, t)$ with respect to the space $\vec{r}$ and time $t$. We considered the parameters $E_{x}=82 \mathrm{meV}, m_{c}=3 \times 10^{-5} m_{e}, \gamma=3 \mathrm{meV}, \alpha_{R}=$ $0.6 \mathrm{meV} \mu \mathrm{m}^{2}, \alpha_{I}=0.2 \mathrm{meV} \mu \mathrm{m}^{2}, \lambda=0.07, k_{p}=6.7 \mu \mathrm{m}^{-1}$, $g=60 \mathrm{meV}$, and $A_{p}=12 \mu \mathrm{m}^{2}$, where $m_{e}$ is the electron mass.

\section{Configuration of the Perovskite Polariton Parametric Oscillator}

In our experiment, the $\mathrm{CsPbr}_{3}$ single-crystal film grown by CVD (Sec. 2) $)^{47,48}$ exhibits a flat surface with strong 
(a)

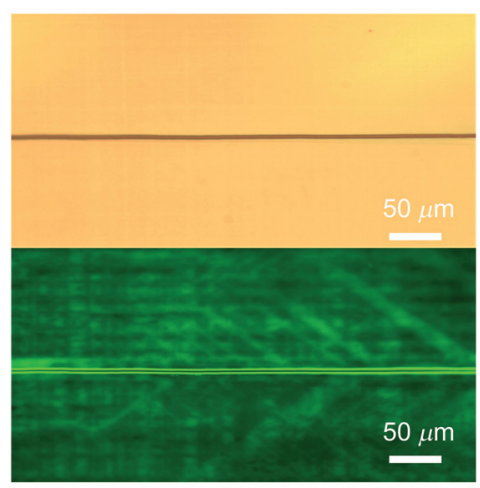

(c)

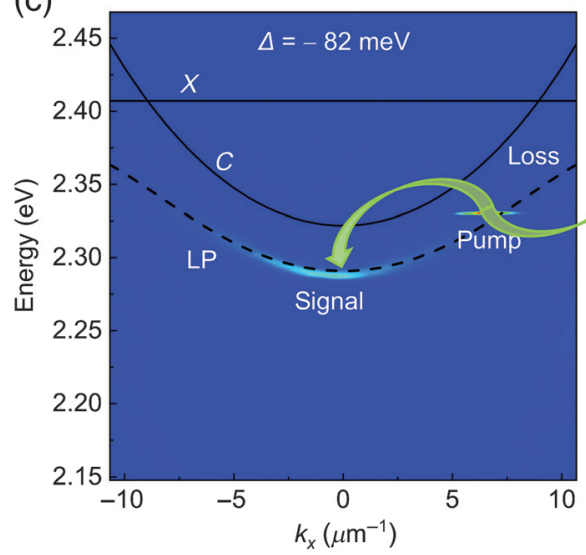

(b)

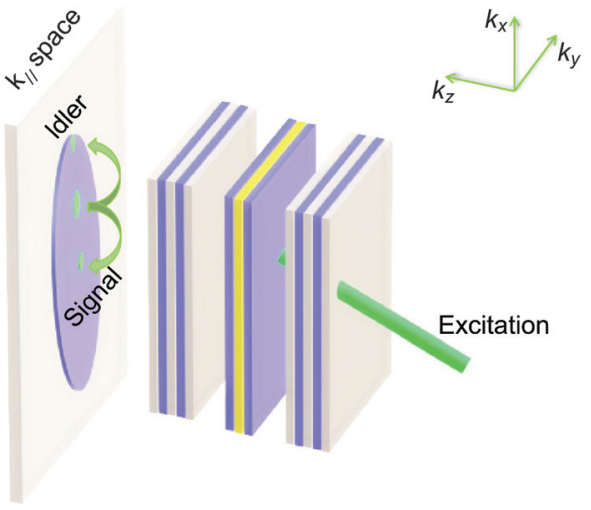

(d)

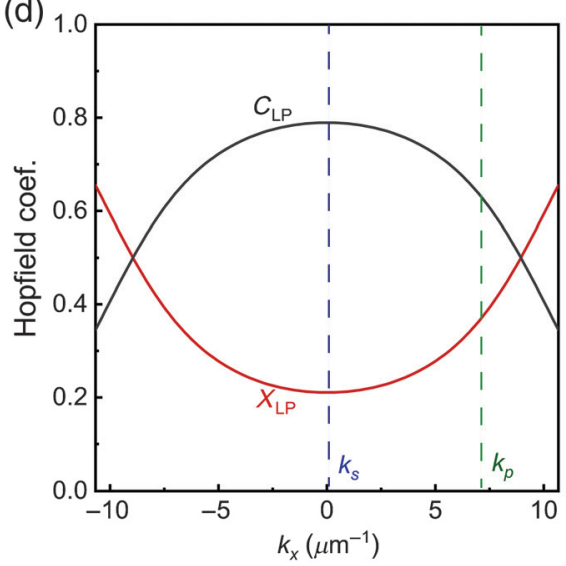

Fig. 1 Schematic diagram and mechanism of polariton parametric oscillator in the perovskite microcavity. (a) Microscopy image and fluorescence microscopy image of the $\mathrm{CsPbBr}_{3}$ perovskite single crystal. (b) Experimental geometry of the $\mathrm{CsPbBr}_{3}$ perovskite microcavity, in which a thick $\mathrm{Cs} \mathrm{PbBr}_{3}$ perovskite is sandwiched by two DBRs. (c) Angle-resolved photoluminescence spectrum of $\mathrm{CsPbBr}_{3}$ microcavity along $\mathrm{H}$ polarization under $\mathrm{CW}$ excitations. The dashed black line displays the theoretical fitting dispersion of the LP dispersion; the solid black lines show the dispersions of uncoupled $\mathrm{CsPbBr}_{3}$ perovskite exciton $(X)$ and cavity photon mode $(C)$; the detuning $\Delta$ is indicated in this figure. (d) Hopfield coefficients illustrating the exciton $\left(X_{\mathrm{LP}}\right)$ and photon $\left(C_{\mathrm{LP}}\right)$ fraction of the LP dispersion along $x$ polarization; the blue vertical line denotes the signal state polariton $\left(k_{s}\right)$; the green vertical line represents the pump state polariton $\left(k_{p}\right)$.

green fluorescence, as shown in Fig. 1(a) (see also Fig. S1 in the Supplementary Material). The geometry of the polariton parametric oscillator is schematically displayed in Fig. 1(b), where the signal and idler can be generated along the $k_{x}$ axis in far-field emission after the $\mathrm{Cs}_{\mathrm{PbBr}}$ microcavity is resonantly excited with a critical (magic) angle along $k_{x}$. The $\mathrm{CsPBr}_{3}$ microcavity itself consists of a $\mathrm{CsPBr}_{3}$ single-crystal film with a thickness of $280 \mathrm{~nm}$ (Fig. S2 in the Supplementary Material), sandwiched between two DBRs deposited by electron beam evaporation (Sec. 2). Figure 1(c) presents the angle-resolved photoluminescence spectrum of the microcavity in horizontal $(H)$ polarization under nonresonant $\mathrm{CW}$ laser $(457 \mathrm{~nm})$ excitation. Using a coupled oscillator model to fit the polariton dispersion obtained in the measurement, we extract a bare exciton energy of $2.407 \mathrm{eV}$, cavity-exciton detuning $\Delta=-82 \mathrm{meV}$, Rabi splitting of $120 \mathrm{meV}$, and refractive index of 2.860 . If this system is excited by a resonant pump laser matching with the polariton dispersion at $k_{p}$, the polariton pairs could scatter and oscillate to the signal state and the high $k$ state (idler), respectively. The intensity of the polariton dispersion shown in Fig. 1(c) is weaker at higher $k$ states. We attribute this to the small photonic fraction of these states as well as their effective losses caused by additional energy relaxation processes. We can also see that the polariton blueshift is larger for higher $k$. This can be attributed to $k$-dependence of the excitonic fraction, where the exciton fraction of the pump state at $k_{p}$ is twice as large as that of the signal state at $k_{s}$. Such nonuniform renormalization of the dispersion has also been discussed theoretically. ${ }^{49}$

\section{Observation and Characterizations of Polariton Oscillation}

The system is further resonantly excited by a pulsed nanosecond laser at $533 \mathrm{~nm}$ to reach the nonlinear regime in the transmission configuration, and the resonant excitation is spectrally tuned to match the high $k$ state $\left(k_{x}=6.8 \mu \mathrm{m}^{-1}\right)$ in the polariton dispersion with a negative detuning of $\Delta=-82 \mathrm{meV}$. At the pump power of $0.5 P_{\text {th }}$, where $P_{\text {th }}$ is the power threshold for 

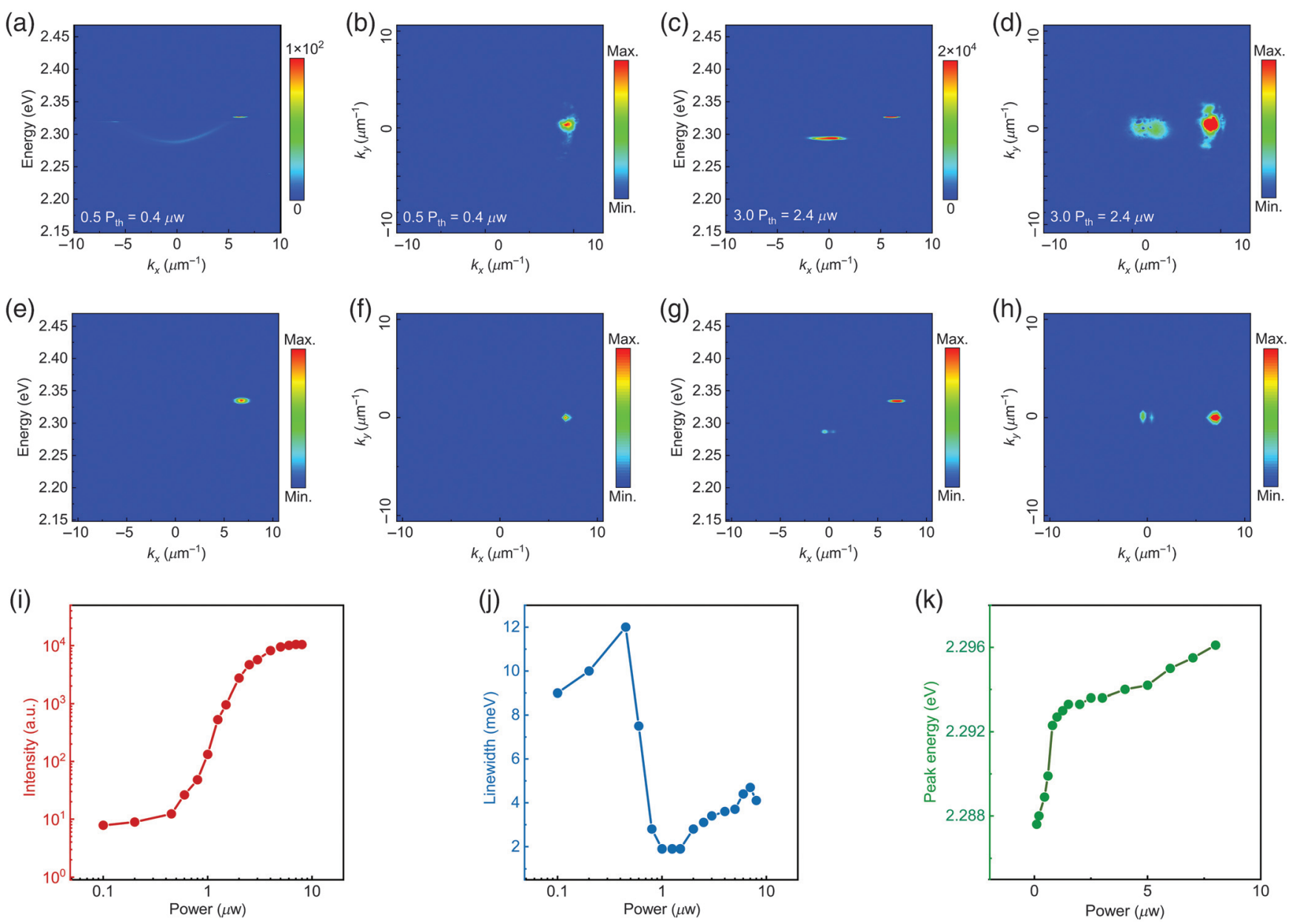

Fig. 2 Observation and characterizations of polariton oscillation at room temperature. Experimental far-field emission of (a) energy $-k_{x}$ and (b) $k_{x}-k_{y}$ at the pump power of $0.5 P_{\text {th }}$. Experimental far-field emission of (c) energy $-k_{x}$ and (d) $k_{x}-k_{y}$ at the pump power of $3 P_{\text {th }}$. Theoretically calculated far-field emission of (e) energy $-k_{x}$ and (f) $k_{x}-k_{y}$ at the pump power of $0.5 P_{\text {th }}$. Theoretically calculated far-field emission of $(\mathrm{g})$ energy $-k_{x}$ and (h) $k_{x}-k_{y}$ at the pump power of $3 P_{\text {th }}$. (i) Signal-state $k_{s}$ emission intensity as a function of pump $k_{p}$ fluence in a log-log scale, demonstrating a super-linear increase by three orders of magnitude near threshold. (j) Signal-state $k_{s}$ emission linewidth as a function of pump $k_{p}$ fluence along with a sharp narrowing linewidth from 12 to $2 \mathrm{meV}$ at the threshold. (k) Signal-state $k_{s}$ emission peak energy with a continuous blueshift trend.

parametric oscillation, the polariton energy dispersion along $k_{x}$ is observed without a clear signal state in Fig. 2(a), in accordance with the dispersions displayed in Fig. 1(c). Similarly, the $k_{x}-k_{y}$ far-field emission [Fig. 2(b)] shows the strong pump state along $k_{x}$. With increasing pump power, polariton scattering becomes more efficient and triggers polariton parametric oscillation, where we observe strong emission from the ground state as the signal state. As shown in Figure 2(c), at the pump power of $3 P_{\text {th }}$, the signal state appears at the ground state of the dispersion, whereas the idler state at $k_{x}=13.6 \mu \mathrm{m}^{-1}$ was not observed (partly since the wavevector of the idler was beyond the collected range). Accordingly, the $k_{x}-k_{y}$ far-field emission exhibits a signal state at $k_{x}=0 \mu \mathrm{m}^{-1}, k_{y}=0 \mu \mathrm{m}^{-1}$ and a pump state at $k_{x}=6.8 \mu \mathrm{m}^{-1}, k_{y}=0 \mu \mathrm{m}^{-1}$. Considering the polariton-polariton nonlinear pump term, the theoretical calculations [Figs. 2(e)-2(h)] are in good agreement with experiments [Figs. 2(a)-2(d)], respectively.
To characterize the occurrence of polariton parametric oscillation quantitatively, we extract the emission intensity, linewidth, and peak energy of the signal state from the power-dependent angle-resolved emission spectra. A superlinear increase of emission intensity by three orders of magnitude is observed near the threshold $P_{\mathrm{th}}=0.8 \mu \mathrm{W}$ [Fig. 2(i)], as well as a sharp linewidth narrowing from 12 to $2 \mathrm{meV}$ [Fig. 2(j)]. Importantly, with increasing pump power, the peak energy of the signal state exhibits a continuous blueshift [Fig. 2(k)], arising from polariton-polariton interaction in the system.

\section{Characterizations of Polariton Oscillator versus Pump States}

To further prove the occurrence of polariton parametric oscillation, we have conducted measurements by changing the incident 

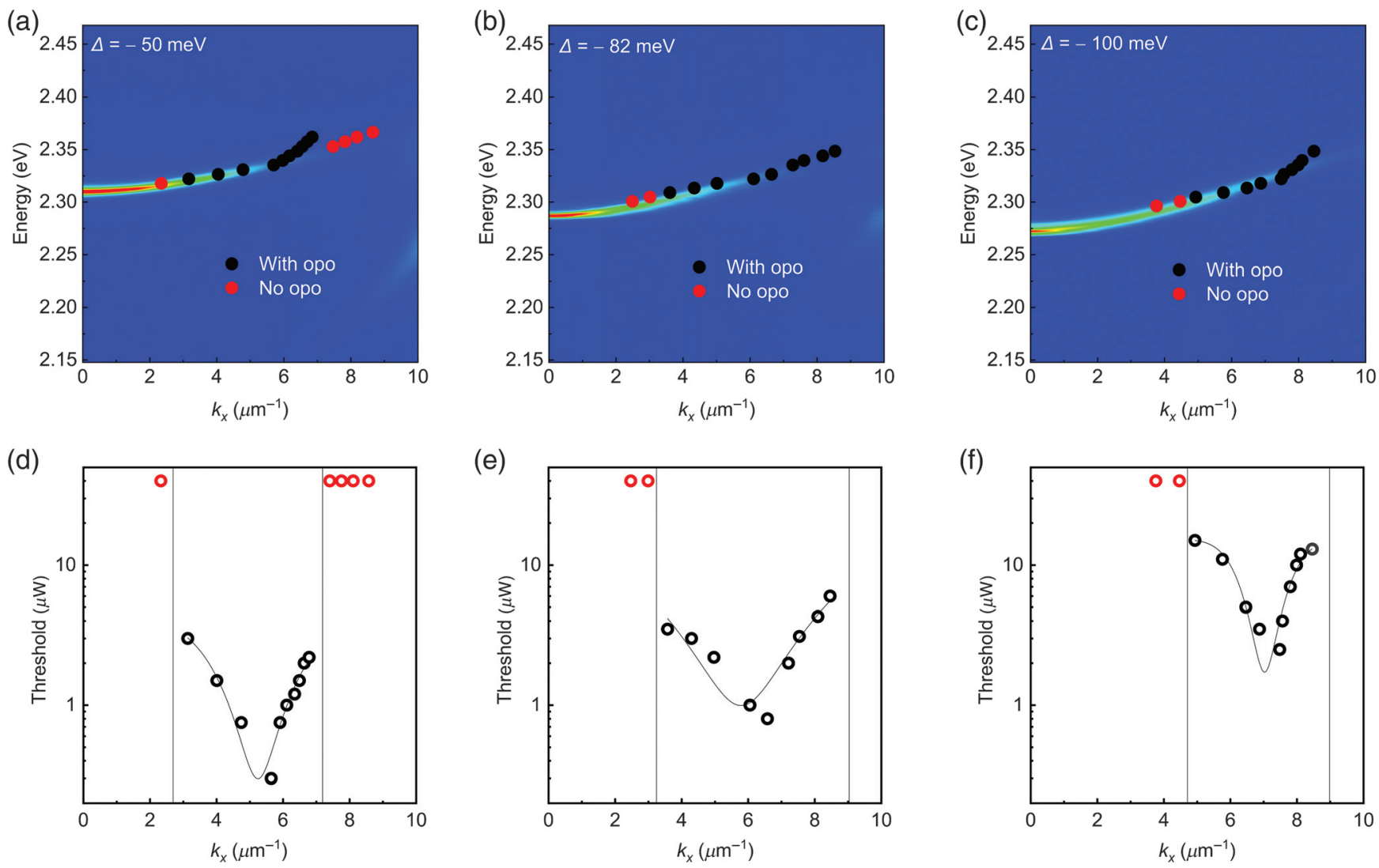

Fig. 3 Characterizations of polariton oscillator versus pump states for three samples with different detunings $\Delta=-50,-82,-100 \mathrm{meV}$, respectively. (a)-(c) The pump state is tuned with energy and angle to resonantly excite the LP dispersion for detunings (a) $\Delta=-50 \mathrm{meV}$, (b) $\Delta=-82 \mathrm{meV}$, and (c) $\Delta=-100 \mathrm{meV}$. (d)-(f) The energy conversion threshold as a function of pump state angle for detunings (d) $\Delta=-50 \mathrm{meV}$, (e) $\Delta=-82 \mathrm{meV}$, and (f) $\Delta=-100 \mathrm{meV}$. The lowest energy conversion threshold peaks at (d) $P_{\text {th }}=0.3 \mu \mathrm{W}$, (e) $P_{\text {th }}=0.8 \mu \mathrm{W}$, and (f) $P_{\text {th }}=2.5 \mu \mathrm{W}$, respectively. The black circles denote the occurrence of OPO, whereas the red circles represent cases where the OPO was not present.

pump state along the polariton dispersion for three different cavity-exciton detunings. Specifically, the wavelength (energy) of the excitation can be changed by the nanosecond pulsed laser in steps of one nanometer precision, while the incident pump angle (momentum) is continuously tuned at each wavelength of the excitation to obtain the maximum intensity with the lowest threshold and in resonance with the LP dispersion. As shown in Figs. 3(a)-3(c), the pump states are resonantly excited, while a slight blueshift is observed between pump states and polariton dispersion at higher $k$, resulting from the higher exciton fraction. The parametric oscillation thresholds as a function of pump states in Figs. 3(a)-3(c) are plotted in Figs. 3(d)-3(f), respectively. A strong reduction of threshold is observed at the critical resonant angle, where phase matching conditions best match the shape of the LP dispersions. Above the resonant critical angle, the threshold increases with the increase of the pump angle. When the resonant pump angle is smaller than the resonant critical angle, the oscillator threshold increases with the decrease of pump angle and is absent approaching normal incidence. Such evidence strongly suggests that the signal is dominantly contributed from parametric oscillation rather than energy relaxation. Furthermore, the critical angle changes with the detuning: $k_{x}=5.7 \mu \mathrm{m}^{-1}$ for detuning $\Delta=-50 \mathrm{meV}$ with $P_{\text {th }}=0.3 \mu \mathrm{W}$ [Figs. 3(a) and 3(d)], $k_{x}=6.8 \mu \mathrm{m}^{-1}$ for detuning $\Delta=-82 \mathrm{meV}$ with $P_{\text {th }}=0.8 \mu \mathrm{W}$ [Figs. 3(b) and 3(e)], and $k_{x}=7.6 \mu \mathrm{m}^{-1}$ for detuning $\Delta=-100 \mathrm{meV}$ with $P_{\text {th }}=$ $2.5 \mu \mathrm{W}$ [Figs. 3(c) and 3(f)], following the principle of larger critical angle for more negative detuning due to the conservation of energy and momentum. In addition, the lowest threshold at critical angle is lower at a less negative detuning as a result of a higher exciton fraction leading to a stronger polariton-polariton interaction, which further implies the appearance of polariton parametric oscillation.

\section{Polarization Dependence of the Polariton Parametric Oscillator}

We further investigate the polarization dependence of the signal in our perovskite microcavity. ${ }^{26,50}$ Under CW laser (457 nm) excitation, due to the intrinsic anisotropy of the perovskite crystal, ${ }^{51}$ the LP dispersion shows a linearly polarized splitting at $k=0, E_{s V}=2.296 \mathrm{eV}$ along $V$ polarization, and $E_{s H}=$ $2.287 \mathrm{eV}$ along $H$ polarization in Fig. 4(a). The sample is oriented with the crystal boundary exhibited in Fig. S1 in the 
(a)

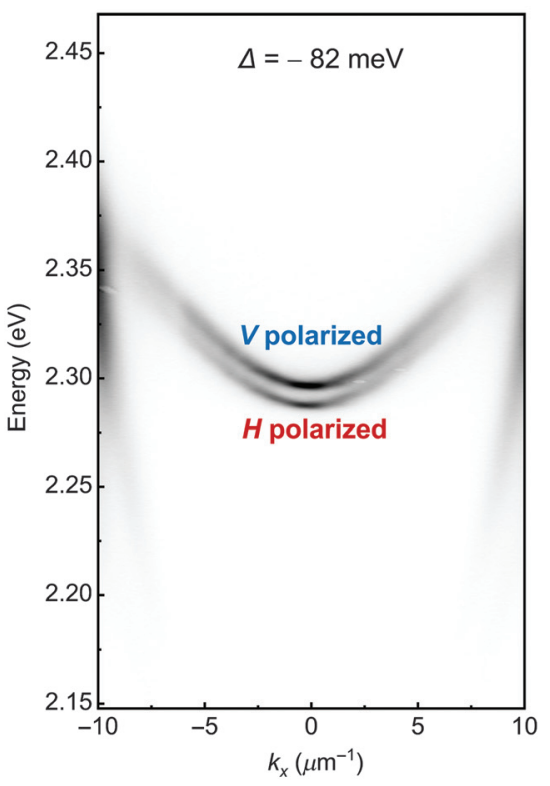

(b)

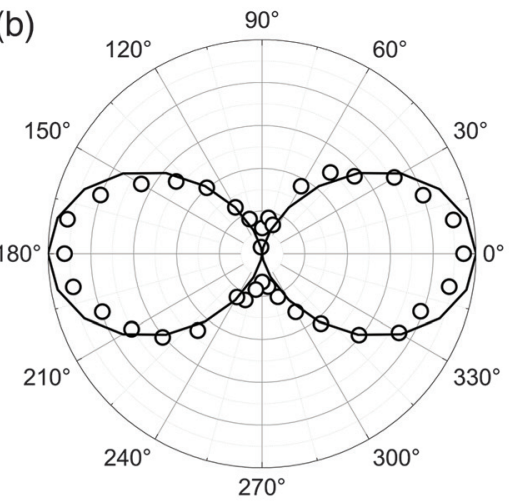

(c)

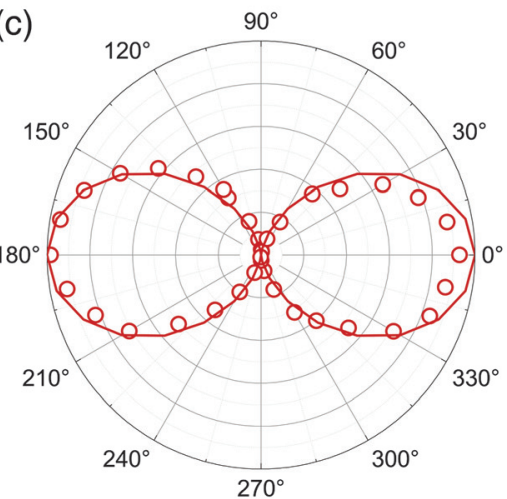

(d)

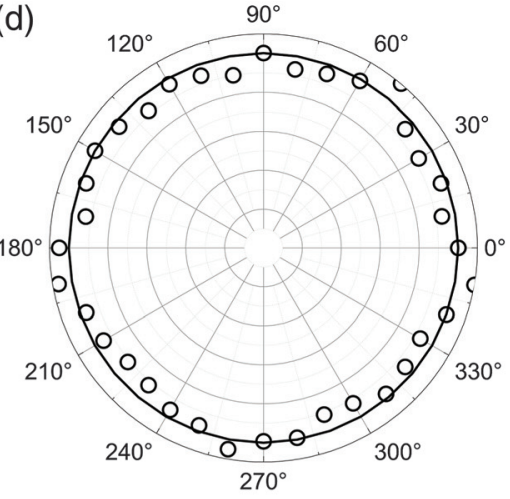

(e)

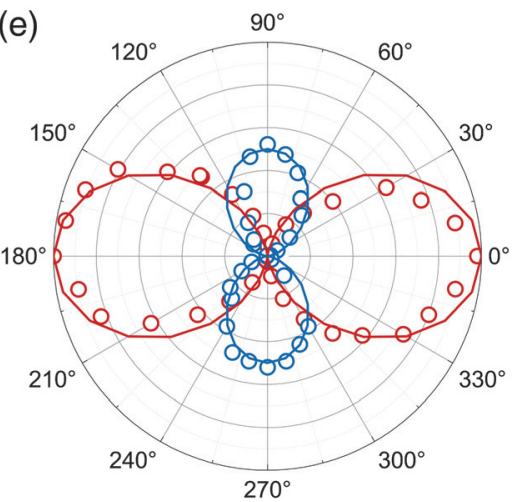

Fig. 4 Polarization dependence of the polariton parametric oscillator. (a) Angle-resolved photoluminescence spectrum of $\mathrm{CsPbBr}_{3}$ microcavity along $V$ polarization and $H$ polarization under $\mathrm{CW}$ excitation. (b) Polar plot of the $H$ polarized pump excited at $E_{p}=2.326 \mathrm{eV}(533 \mathrm{~nm})$, centered $k_{x}=6.8 \mu \mathrm{m}^{-1}$ (black circle dots), and fitting function (black solid line) $f \propto \cos ^{2} \theta$. (c) Under excitation of (b), polar plot of the measured polarization emission of the signal state at $E_{s H}=2.287 \mathrm{eV}, k_{x}=0 \mu \mathrm{m}^{-1}$ (red circle dots) and fitting function (red solid line) $f \propto \cos ^{2} \theta$. (d) Polar plot of the left circularly polarized pump excited at $E_{p}=2.326 \mathrm{eV}(533 \mathrm{~nm})$, centered $k_{x}=6.8 \mu \mathrm{m}^{-1}$ (black circle dots), as well as a fitting constant function (continuous line). (e) Under excitation of (d), polar plot of the measured polarization emission of the signal state at $E_{s H}=2.287 \mathrm{eV}, k_{x}=0 \mu \mathrm{m}^{-1}$ (red circle dots), and fitting function (red solid line) $f \propto \cos ^{2} \theta$; at $E_{s V}=2.296 \mathrm{eV}, k_{x}=0 \mu \mathrm{m}^{-1}$ (blue circle dots), and fitting function (blue solid line) $f \propto \cos ^{2}(\theta+90 \mathrm{deg})$.

Supplementary Material, so the linear polarization direction coincides with the crystal axes. For characterizing the polarization dependence of the signal in the perovskite polariton oscillator, the polarization of the signal is explored under a linearly polarized pump and circularly polarized pump, respectively. Figure 4(b) displays the high $H$ linearly polarized pump after the DBR microcavity. Under this $H$ polarized pump at critical point $\left[E_{p}=2.326 \mathrm{eV}(533 \mathrm{~nm}), k_{x}=6.8 \mu \mathrm{m}^{-1}\right]$, the polar plot of the signal is detected and fitted by Malus's law, showing high $H$ linear polarization as well [Fig. 4(c)]. Furthermore, the system is excited by a left circular pump [Fig. 4(d)] near the critical angle $\left[E_{p}=2.326 \mathrm{eV}(533 \mathrm{~nm}), k_{x}=6.8 \mu \mathrm{m}^{-1}\right]$, where the pump in $k$ space is wide enough to cover the $H$ polarized polariton mode and $V$ polarized polariton mode simultaneously. The $H$ linearly polarized signal at $E_{s H}=2.287 \mathrm{eV}$ or $V$ linearly polarized signal at $E_{s V}=2.296 \mathrm{eV}$ [Fig. 4(e)] with respective blueshifts is observed, rather than inheriting the circular polarization of the pump, corresponding with the states noted in Fig. 4(a). The pump angle is the critical (magic) angle for the $H$ polariton mode, while slightly larger than that for $V$ polariton mode, thus the intensity of the $H$ polarized signal is found to be about two times stronger than that of the $V$ polarized signal in Fig. 4(e). These linearly polarized signal dependences correspond with the strong linear anisotropy in the perovskite system that could split the polarization of excitation into linearly polarized components.

\section{Conclusions and Discussions}

By implementing resonant excitation at a critical (magic) angle, we experimentally and theoretically report the nondegenerate polariton parametric oscillator in perovskite exciton-polaritons at room temperature. The result corresponds to spontaneous parametric down conversion over a range of angles. Meanwhile, the signal state is split into two nondegenerate orthogonal linear polarizations due to the strong anisotropy of perovskite. Our work reveals an alternative avenue for lower threshold nonlinear polaritonic devices. 


\section{Acknowledgments}

Q. X. gratefully acknowledges the National Natural Science Foundation of China (No. 12020101003), the State Key Laboratory of Low-Dimensional Quantum Physics for the strong support, and Tsinghua University for the start-up grant. J. Q. W., S. G., R. S., A. F., and T. C. H. L. acknowledge the support from the Singapore Ministry of Education via the AcRF Tier 3 Programme "Geometrical Quantum Materials" (No. MOE2018-T3-1-002) and AcRF Tier 2 (Nos. MOE2018T2-2-068 and MOE2019-T2-1-004). The authors declare no competing interests.

\section{References}

1. J. A. Giordmaine and R. C. Miller, "Tunable coherent parametric oscillation in $\mathrm{LiNbO}_{3}$ at optical frequencies," Phys. Rev. Lett. 14(24), 973-976 (1965).

2. L.-A. Wu et al., "Generation of squeezed states by parametric down conversion," Phys. Rev. Lett. 57(20), 2520-2523 (1986).

3. H. Jabri and H. Eleuch, "Interaction of a dipolariton system with squeezed light from a parametric down-conversion process," Phys. Rev. A 101(5), 053819 (2020).

4. M. Förtsch et al., "A versatile source of single photons for quantum information processing," Nat. Commun. 4(1), 1818 (2013).

5. X. Guo et al., "Parametric down-conversion photon-pair source on a nanophotonic chip," Light Sci. Appl. 6(5), e16249 (2017).

6. X. Lu et al., "Chip-integrated visible-telecom entangled photon pair source for quantum communication," Nat. Phys. 15(4), 373-381 (2019).

7. T. Inagaki et al., "Large-scale Ising spin network based on degenerate optical parametric oscillators," Nat. Photonics 10(6), 415-419 (2016).

8. A. W. Bruch et al., "On-chip $\chi^{(2)}$ microring optical parametric oscillator," Optica 6(10), 1361-1366 (2019).

9. I. Breunig, "Three-wave mixing in whispering gallery resonators," Laser Photonics Rev. 10(4), 569-587 (2016).

10. Z.-G. Lin, L.-C. Tang, and C.-P. Chou, "Characterization and properties of novel infrared nonlinear optical crystal $\mathrm{CsGe}\left(\mathrm{Br}_{x} \mathrm{Cl}_{1-x}\right)_{3}$," Inorg. Chem. 47(7), 2362-2367 (2008).

11. J. Xu et al., "Halide perovskites for nonlinear optics," Adv. Mater. 32(3), 1806736 (2020).

12. Y. Zhou et al., "Nonlinear optical properties of halide perovskites and their applications," Appl. Phys. Rev. 7(4), 041313 (2020).

13. T. J. Kippenberg, S. M. Spillane, and K. J. Vahala, "Kerrnonlinearity optical parametric oscillation in an ultrahigh- $Q$ toroid microcavity," Phys. Rev. Lett. 93(8), 083904 (2004).

14. J. U. Fürst et al., "Low-threshold optical parametric oscillations in a whispering gallery mode resonator," Phys. Rev. Lett. 105(26), 263904 (2010).

15. T. Beckmann et al., "Highly tunable low-threshold optical parametric oscillation in radially poled whispering gallery resonators," Phys. Rev. Lett. 106(14), 143903 (2011).

16. N. L. B. Sayson et al., "Octave-spanning tunable parametric oscillation in crystalline Kerr microresonators," Nat. Photonics 13(10), 701-706 (2019).

17. X. Lu et al., "Milliwatt-threshold visible-telecom optical parametric oscillation using silicon nanophotonics," Optica 6(12), 15351541 (2019).

18. J. Lu et al., "Ultralow-threshold thin-film lithium niobate optical parametric oscillator," Optica 8(4), 539-544 (2021).

19. C. Trovatello et al., "Optical parametric amplification by monolayer transition metal dichalcogenides," Nat. Photonics 15(1), 6-10 (2021).

20. H. Deng, H. Haug, and Y. Yamamoto, "Exciton-polariton BoseEinstein condensation," Rev. Mod. Phys. 82(2), 1489-1537 (2010).
21. T. Byrnes, N. Y. Kim, and Y. Yamamoto, "Exciton-polariton condensates," Nat. Phys. 10(11), 803-813 (2014).

22. D. Sanvitto and S. Kéna-Cohen, "The road towards polaritonic devices," Nat. Mater. 15(10), 1061-1073 (2016).

23. J. J. Baumberg et al., "Parametric oscillation in a vertical microcavity: a polariton condensate or micro-optical parametric oscillation," Phys. Rev. B 62(24), R16247-R16250 (2000).

24. P. G. Savvidis et al., "Angle-resonant stimulated polariton amplifier," Phys. Rev. Lett. 84(7), 1547-1550 (2000).

25. M. Saba et al., "High-temperature ultrafast polariton parametric amplification in semiconductor microcavities," Nature 414(6865), 731-735 (2001).

26. G. Dasbach et al., "Polarization inversion via parametric scattering in quasi-one-dimensional microcavities," Phys. Rev. B 71(16), 161308 (2005).

27. C. Diederichs et al., "Parametric oscillation in vertical triple microcavities," Nature 440(7086), 904-907 (2006).

28. A. S. Kuznetsov et al., "Dynamically tuned arrays of polariton parametric oscillators," Optica 7(12), 1673-1681 (2020).

29. V. Ardizzone et al., "Bunching visibility of optical parametric emission in a semiconductor microcavity," Phys. Rev. B 86(4), 041301 (2012).

30. T. Lecomte et al., "Optical parametric oscillation in onedimensional microcavities," Phys. Rev. B 87(15), 155302 (2013).

31. W. Xie et al., "Room-temperature polariton parametric scattering driven by a one-dimensional polariton condensate," Phys. Rev. Lett. 108(16), 166401 (2012).

32. J. Wu et al., "Nonlinear parametric scattering of exciton polaritons in perovskite microcavities," Nano Lett. 21(7), 3120-3126 (2021).

33. L. N. Quan et al., "Perovskites for light emission," Adv. Mater. 30(45), 1801996 (2018).

34. J. Xing et al., "High-efficiency light-emitting diodes of organometal halide perovskite amorphous nanoparticles," ACS Nano 10(7), 6623-6630 (2016).

35. S. A. Veldhuis et al., "Perovskite materials for light-emitting diodes and lasers," Adv. Mater. 28(32), 6804-6834 (2016).

36. Q. Zhang et al., "Halide perovskite semiconductor lasers: materials, cavity design, and low threshold," Nano Lett. 21(5), 1903-1914 (2021).

37. M. Ahmadi, T. Wu, and B. Hu, "A review on organic-inorganic halide perovskite photodetectors: device engineering and fundamental physics," Adv. Mater. 29(41), 1605242 (2017).

38. K. N. Krishnakanth et al., "Broadband femtosecond nonlinear optical properties of $\mathrm{CsPbBr}_{3}$ perovskite nanocrystals," Opt. Lett. 43(3), 603-606 (2018).

39. F. Zhou et al., "2D perovskites with giant excitonic optical nonlinearities for high-performance sub-bandgap photodetection," Adv. Mater. 31(48), 1904155 (2019).

40. L. Wu et al., "Perovskite $\mathrm{CsPbX}_{3}$ : a promising nonlinear optical material and its applications for ambient all-optical switching with enhanced stability," Adv. Opt. Mater. 6(19), 1800400 (2018).

41. R. Su et al., "Room-temperature polariton lasing in all-Inorganic perovskite nanoplatelets," Nano Lett. 17(6), 3982-3988 (2017).

42. R. Su et al., "Room temperature long-range coherent exciton polariton condensate flow in lead halide perovskites," Sci. Adv. 4(10), eaau0244 (2018).

43. R. Su et al., "Perovskite semiconductors for room-temperature exciton-polaritonics," Nat. Mater. 20(10), 1315-1324 (2021).

44. R. Su et al., "Optical switching of topological phase in a perovskite polariton lattice," Sci. Adv. 7(21), eabf8049 (2021).

45. A. Fieramosca et al., "Two-dimensional hybrid perovskites sustaining strong polariton interactions at room temperature," Sci. Adv. 5(5), eaav9967 (2019).

46. M. Romanelli et al., "Four wave mixing oscillation in a semiconductor microcavity: generation of two correlated polariton populations," Phys. Rev. Lett. 98(10), 106401 (2007). 
47. S. T. Ha et al., "Synthesis of organic-inorganic lead halide perovskite nanoplatelets: towards high-performance perovskite solar cells and optoelectronic devices," Adv. Opt. Mater. 2(9), 838-844 (2014).

48. Q. Zhang et al., "High-quality whispering-gallery-mode lasing from cesium lead halide perovskite nanoplatelets," Adv. Funct. Mater. 26(34), 6238-6245 (2016).

49. D. M. Whittaker, "Effects of polariton-energy renormalization in the microcavity optical parametric oscillator," Phys. Rev. B 71(11), 115301 (2005).

50. P. G. Lagoudakis et al., "Stimulated spin dynamics of polaritons in semiconductor microcavities," Phys. Rev. B 65(16), 161310 (2002).

51. R. Su et al., "Observation of exciton polariton condensation in a perovskite lattice at room temperature," Nat. Phys. 16(3), 301-306 (2020).

Jinqi Wu is a PhD candidate at the School of Physical and Mathematical Sciences, Nanyang Technological University. Her major research interest is in the field of nonlinearity of exciton-polaritons in perovskite.

Rui Su received his PhD at the School of Physical and Mathematical Sciences, Nanyang Technological University in 2019 . He is currently a research fellow in the same university and his research efforts focus on exciton polaritons with perovskites.

Antonio Fieramosca received his MSc degree in nanotechnology engineering from University La Sapienza in 2013 and his PhD from Università del Salento in 2019. He is currently a research fellow at the School of Physical and Mathematical Sciences, Nanyang Technological University, and his research focuses on the advanced optical studies of polariton propagation and nonlinearities in organic, inorganic, and hybrid systems, as well as on the fabrication and engineering of photonic micro/nano structures.

Sanjib Ghosh received his $\mathrm{PhD}$ in physics from the National University of Singapore, Singapore, in 2017. Subsequently, he was a research fellow at NTU, Singapore. He is currently appointed as an assistant research scientist at BAQIS, China. His research focuses on the theoretical side of exciton polaritons, quantum optics, machine learning, and quantum transport in strongly disordered systems.

Jiaxin Zhao received her $\mathrm{PhD}$ in 2021 at the School of Physical and Mathematical Sciences, Nanyang Technological University. She is currently a research fellow in the same university and her research efforts focus on exciton polaritons with transition metal dichalcogenides.

Timothy C. H. Liew is an associate professor at the School of Physical and Mathematical Sciences, Nanyang Technological University. He leads a theoretical research group working on nonlinear photonics, quantum optics, quantum neural networks, and exciton-polaritons.

Qihua Xiong is a professor in the Department of Physics at Tsinghua University. His research focuses on optical spectroscopy and ultrafast spectroscopy for condensed matter systems, including quantum physical properties emerging from quantum confinement and anisotropy in low-dimensional quantum materials, strong light-matter coupling in such materials, light-induced phenomena, and new functionalities in photonics and optoelectronics. 